\title{
Simulation and Experimental Study on Load-bearing Deformation Characteristics of 11R22.5 Vehicle Retreaded Tire
}

\author{
Qiang WANG *, Li JIANG, Xiaojie QI \\ School of Automobile and Traffic Engineering, Heilongjiang Institute of Technology, Harbin, China
}

*Corresponding Author: Qiang WANG ,E-mail: 630702666@qq.com

\begin{abstract}
:
The finite element bearing deformation simulation was implemented on 11.00R22.5 retreaded tires by ANSYS software in the paper in order to further clarify the bearing deformation characteristics of retreaded tires and improve the performance of retreaded tires effectively. The characteristic laws of bearing radial deformation and bearing lateral deformation of retreaded tire and new tires of the same model under different working conditions were obtained through load deformation tests. The radial deformation calculation results, simulation results and measured results of retreaded tires were comparatively analyzed. The calculation formula of bearing radial deformation of retreaded tires was proposed based on the linear regression principle. The difference of bearing deformation characteristics and ground area characteristics of retreaded tires and new tires were comparatively analyzed. The results showed that the radial and lateral deformation of retreaded tires and new tires is increased with the increase of radial load when the tire pressure was constant, and the increase trend is approximately linear. The radial stiffness of retreaded tires is similar to that of new tires under certain tire pressure and low load. The radial stiffness of retreaded tires is larger than that of new tires, and the stiffness difference is increased with the increasing of load under constant tire pressure and high load. Rubber aging phenomenon in retreaded tire carcass have an impact on the bearing deformation characteristics of retreaded tires, thereby producing great impact on the remaining service life of retreaded tires.
\end{abstract}

Keywords: vehicle retreaded tire; load-bearing deformation characteristics; finite element; radial stiffness; rubber aging

\section{Introduction}

The tire industry has undergone rapid development with the rapid development of the automobile industry in China. China has become the largest tire production and consumption power all over the world. However, natural rubber resources are extremely scarce in China, and about $70 \%$ of the annual demand should be imported. Therefore, how to recycle, reduce and make waste tire harmless is related to environmental protection- an important social issue on the one hand, it also belongs to an important topic related to the sustainable development of national economy in China. The waste tires in China are recycled and mainly used to produce recycled rubber and rubber powder. The international practice of "refurbishing followed by scrapping" is usually adopted in order to maximize the effectiveness of waste tire utilization. About $30 \%$ of the economic use value of the whole tires is used only, and about $70 \%$ value can be reused by retreading when the tread is polished ${ }^{[1,15,17]}$. A tire can be retread for many times under sound use and maintenance. The service life of new tires equal to $60 \% \sim 90 \%$ can be restored after each retreading. The total service life of the tire can be extended by $1 \sim 2$ times by repeated retreading. The value of rubber and other resources consumed in the retreading of a waste tire is equivalent to $15 \% \sim 30 \%$ of the production of a new tire, and the sales price is $20 \% \sim 50 \%$ of that of new tires by comparison. Meanwhile, the excellent retreaded tire can even achieve similar mileage and security as that of a new tire. Therefore, we can make the best use of the usage value of tires from retreaded tires. The tire retreading industry can be developed with excellent social and economic benefit as well as great social sustainable development significance $[14,2,11]$. Truck vehicle tires are mostly used in professional transportation systems among various tires, which are characterized by heavy bearing and poor working environment. They are often driven in poor roads and bad environment, which are usually replaced once 1 2 years in batches mostly. Transportation enterprises can save huge cost if retreaded tires are used instead of new tires. However, tire retreading technology is weak in China. The truck tire refurbishment products have short service, thereby greatly limiting promotion and application thereof in any medium, provided the original work is properly cited. 
in a wide range. It is necessary to study the performance of radial retreaded tires of trunks in order to improve the performance and service life of retreaded tires accordingly, thereby laying an important theoretical foundation for optimizing tire retreading process and improving retreaded tire performance ${ }^{[12,13]}$.

\section{Finite element simulation of load-bearing de- formation}

The radial deformation cloud image of 11.00R22.5 retreaded tires is obtained by ANSYS finite element software as shown in figure 1. The lateral deformation cloud image is shown in figure 2. Figure 1 and figure 2 show that the tread of retreaded tires is in full contact with the surface of the ground target near the center of the tire ground mark when the load is $30 \mathrm{kN}$ and the tire pressure is $830 \mathrm{kPa}$, the tread layer becomes flat, and the tire expands outwards laterally. The maximum radial deformation of retreaded tires is $23.72 \mathrm{~mm}$, and the maximum lateral deformation is $23.059 \mathrm{~mm}$. The radial deformation under different loads $(15 \mathrm{kN} \sim 35 \mathrm{kN})$ and different tire pressures $(530 \mathrm{kPa} \sim 830 \mathrm{kpa})$ is obtained as shown in figure 3 , and the lateral deformation is shown in figure 4 through several simulations. Figure 3 and figure 4 show that the radial and lateral deformation of retreaded tires is increased with the

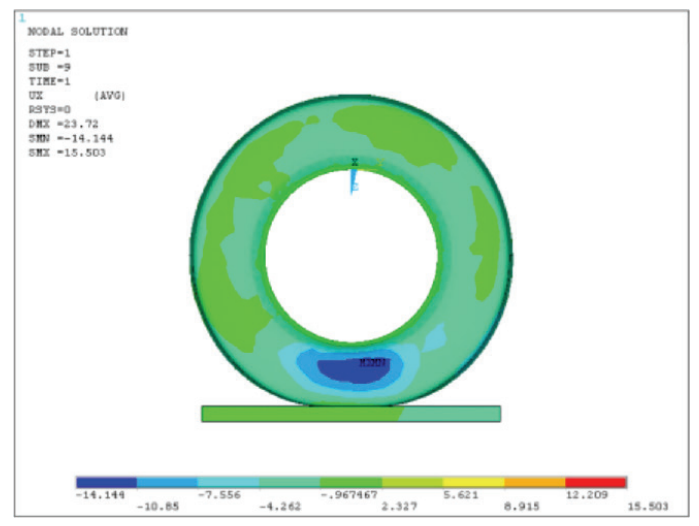

Figure 1 Radial Deformation Graph

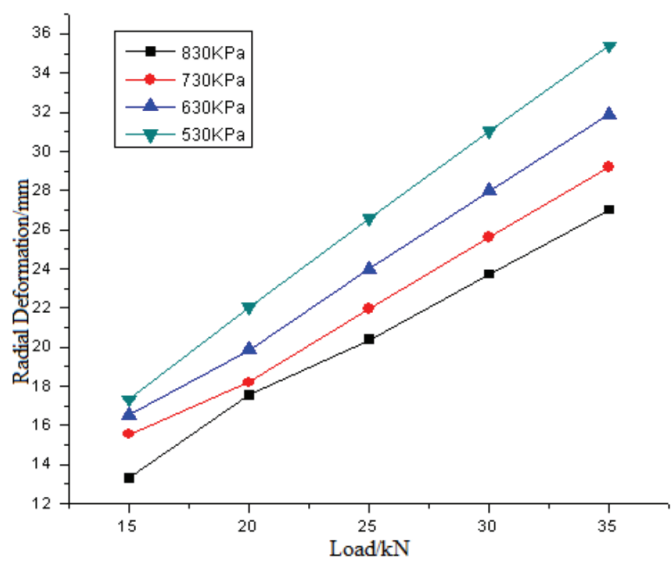

Figure 3 Radial Deformation under different working conditions increase of load when the tire pressure is constant, and the increase trend is approximately linear. The radial and lateral deformation of retreaded tire is increased with the decrease of tire pressure when the load is constant, and the increase trend is also approximately linear. The simulation value of retreaded tire radial deformation is compared with the calculated value when the tire pressure is $530 \mathrm{kPa}$, $630 \mathrm{kPa}, 730 \mathrm{kPa}$ and $830 \mathrm{kPa}$ as shown in figure 5 . Figure 5 shows that the simulation results thereof are constant with the theoretical calculation results under four different tire pressure conditions. The simulation values are slightly larger than the theoretical calculation values mainly because the finite element model has certain calculation accuracy error ${ }^{[7,10]}$.

\section{Load-bearing deformation test}

\subsection{Test purpose}

The radial and lateral deformation of $11.00 \mathrm{R} 22.5$ retreaded tired and new tires of the same model under3.1.1 and3.1.2 working conditions were determined. The bearing deformation laws of retreaded tires and new tires were obtained. The differences thereof were compared. The accuracy of the finite element simulation analysis results was verified $^{[4,5,8]}$.

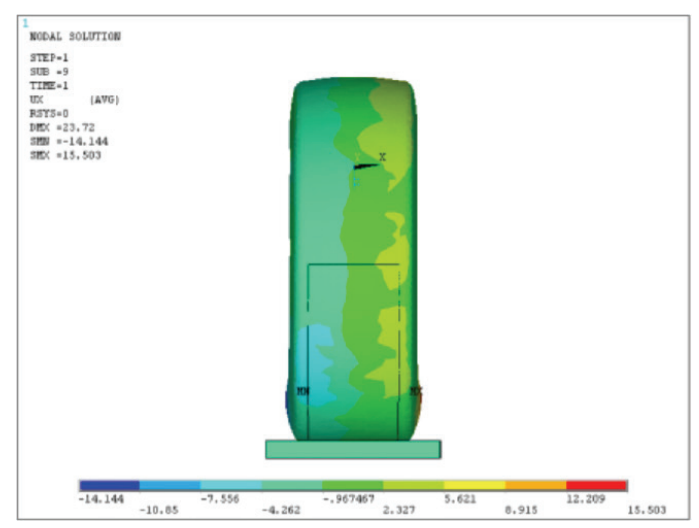

Figure 2 Lateral Deformation Graph

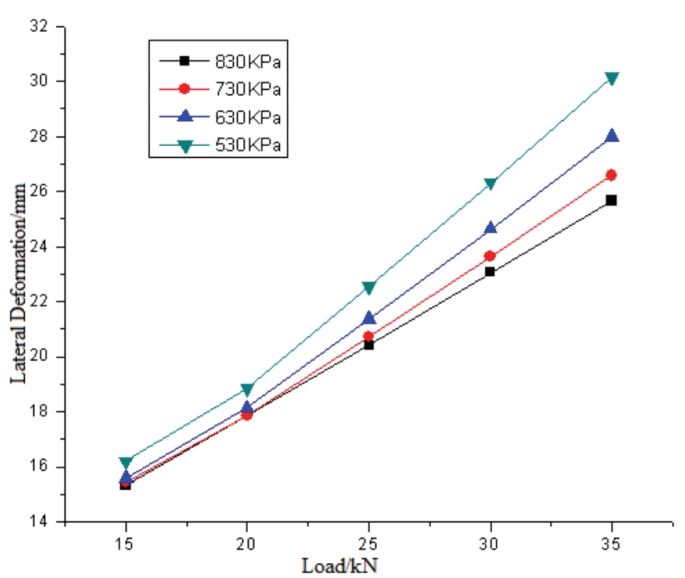

Figure 4 Lateral Deformation under Different Working Conditions 


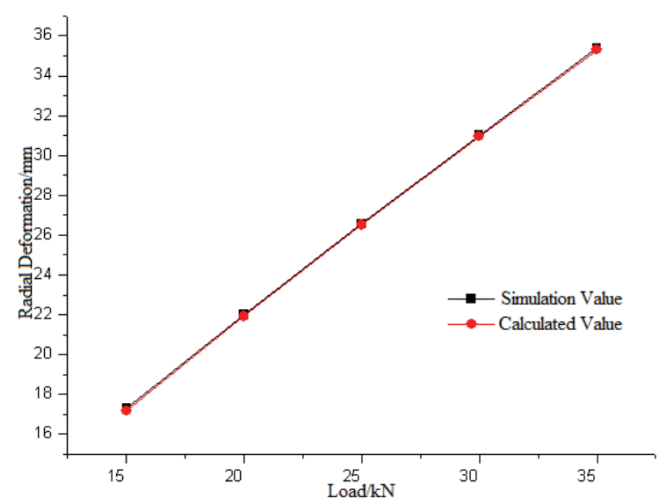

(a) $530 \mathrm{kPa}$

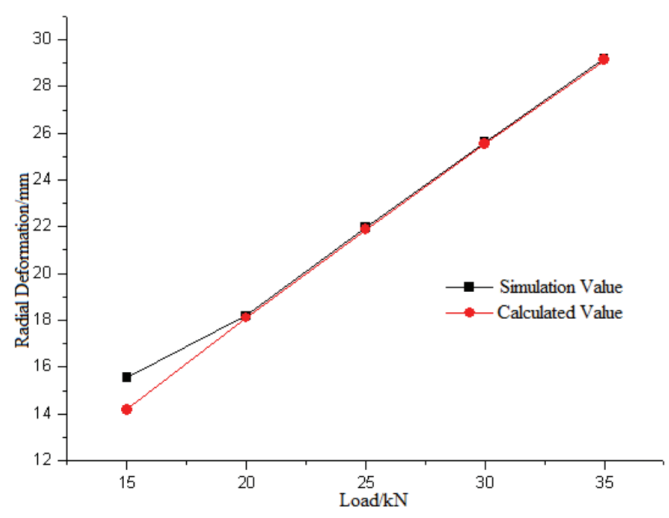

(c) $730 \mathrm{kPa}$

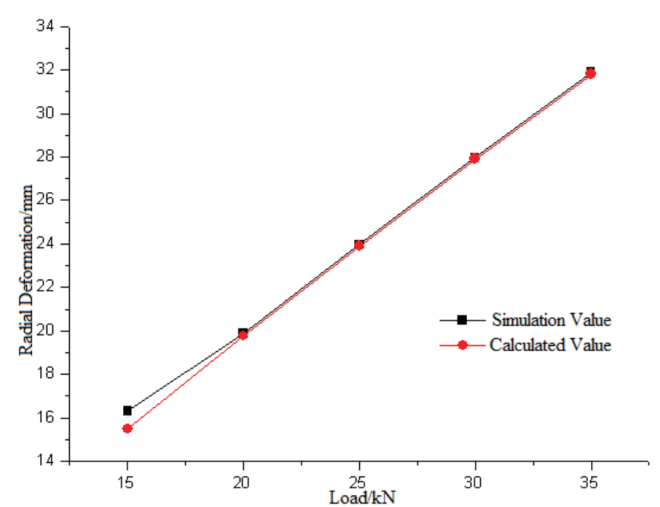

(b) $630 \mathrm{kPa}$

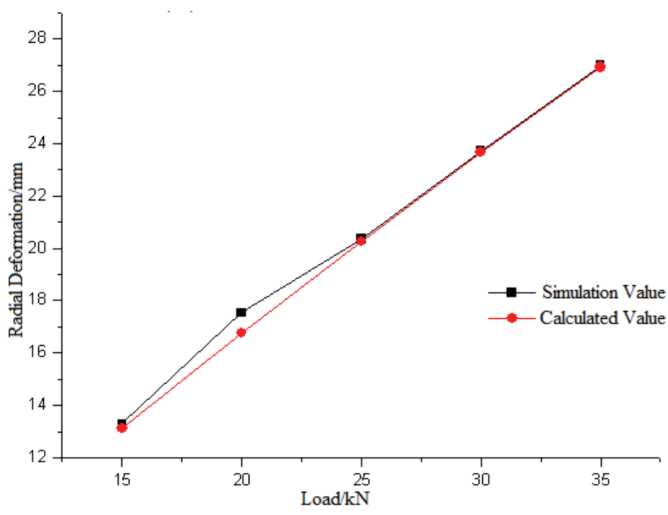

(d) $830 \mathrm{kPa}$

Figure 5 Comparison of Simulation Value and Calculated Value of Radial Deformation under Different Tire Pressures

\subsubsection{Constant tire pressure and load change}

The tire pressure is set as $530 \mathrm{kPa}, 630 \mathrm{kPa}, 730 \mathrm{kPa}$ and $830 \mathrm{kPa}$ respectively. The load $\mathrm{F} 1$ is set as $15 \mathrm{kN}, 20 \mathrm{kN}$, $25 \mathrm{kN}, 30 \mathrm{kN}$ and $35 \mathrm{kN}$. The corresponding loading force $\mathrm{F} 2$ is $30 \mathrm{kN}, 40 \mathrm{kN}, 50 \mathrm{kN}, 60 \mathrm{kN}$ and $70 \mathrm{kN}$.

3.1.2 Constant load and tire pressure change

The load F1 is set as $15 \mathrm{kN}, 20 \mathrm{kN}, 25 \mathrm{kN}, 30 \mathrm{kN}$ and $35 \mathrm{kN}$ respectively. The corresponding loading force $\mathrm{F} 2$ is $30 \mathrm{kN}$, $40 \mathrm{kN}, 50 \mathrm{kN}, 60 \mathrm{kN}$ and $70 \mathrm{kN}$. The tire pressure is set as $530 \mathrm{kPa}, 630 \mathrm{kPa}, 730 \mathrm{kPa}$ and $830 \mathrm{kPa}$.

\subsection{Test system}

The load deformation measurement system of retreaded tires is shown in figure 6 . The test

system is mainly composed of a loading system and a deformation measurement system. The test system belongs to a fore closed loop structure with smooth stress state. The loading force value accuracy error is less than $2 \%$. The main equipment includes press, radial scale, lateral scale, plate, bracket, working platform, axle tube, tire gauge, air compressor, etc. Wherein, a professional production press with an indicating pressure gauge is mainly utilized in the loading system, which is loaded through the hydraulic cylinder manual lever. The loading force range is $0 \sim 30 \mathrm{t}$. The deformation measurement system is mainly composed of a radial scale and

a lateral scale. The measuring accuracy is $\pm 0.5 \mathrm{~mm}^{[9,16]}$.

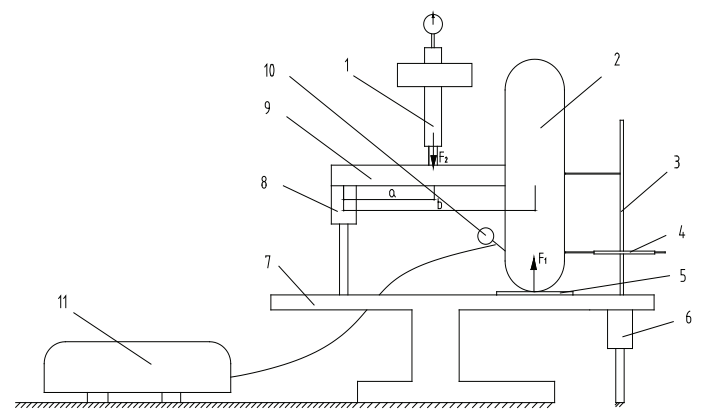

1-A Pressure Machine 2-Tested Tire 3-Radial Ruler 4-Lateral Ruler 5-flat 6-Bracket 1 7-Work Platform 8-Bracket 2 9-Axle Casing 10-Tire Pressure Gauge 11-Air Compressor

Figure 6 Load-bearing Deformation Test System

\section{Results and analysis}

The radial deformation and lateral deformation of retreaded tire under different working conditions is obtained by bearing deformation tests as shown in figure 7 . The radial deformation and lateral deformation of new tire under different working conditions are obtained as shown in figure 8. Figure 7 and figure 8 show that the radial and lateral deformation of retreaded tires and new tires are increased with the increase of vertical load when the tire pressure is constant, and the increase trend is approximately linear. 
It is consistent with the simulation results. The calculated value, simulation value and measured value of retreaded tire radial deformation are compared as shown in figure 9 when the tire pressure is constant. Figure 9 shows that the test values, simulation values and numerical values of retreaded tire radial and lateral deformation are constant well, and the error is less than $3 \%$. The error is produced

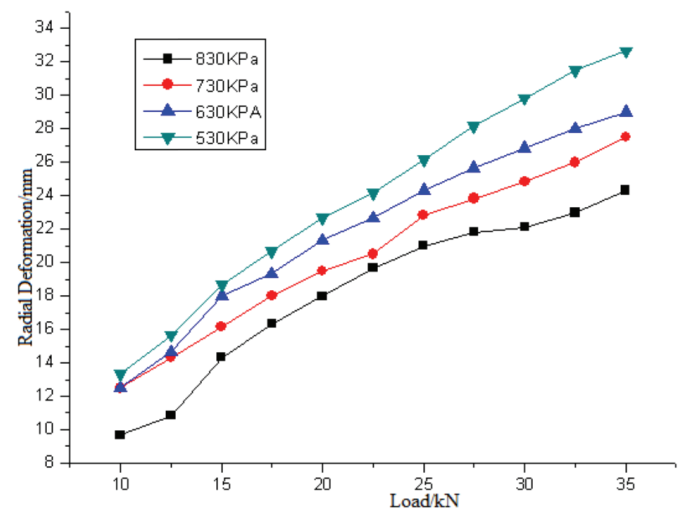

(a) Radial Deformation mainly because of certain precision errors between the loading system and the measurement system. The radial deformation test data are linearly regressed according to the test results, simulation results and numerical calculation results. The obtained linear equation of the radial deformation regression ${ }^{[3,7]}$ is shown in table 1 .

Figure 7 Deformation of Retreaded Tire under Different Conditions

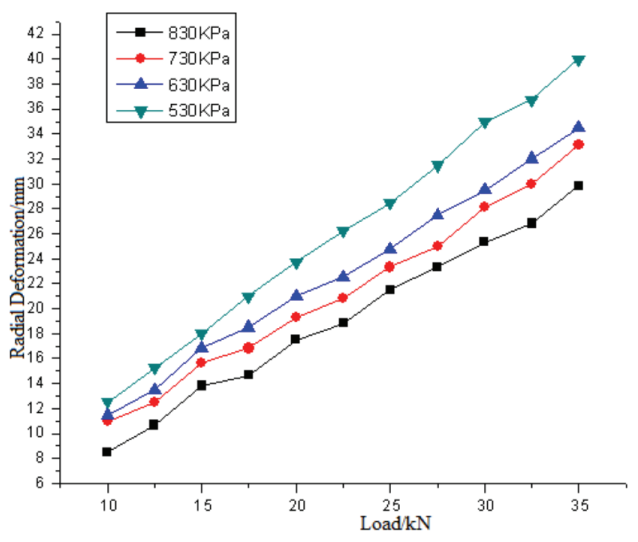

(a) Radial Deformation

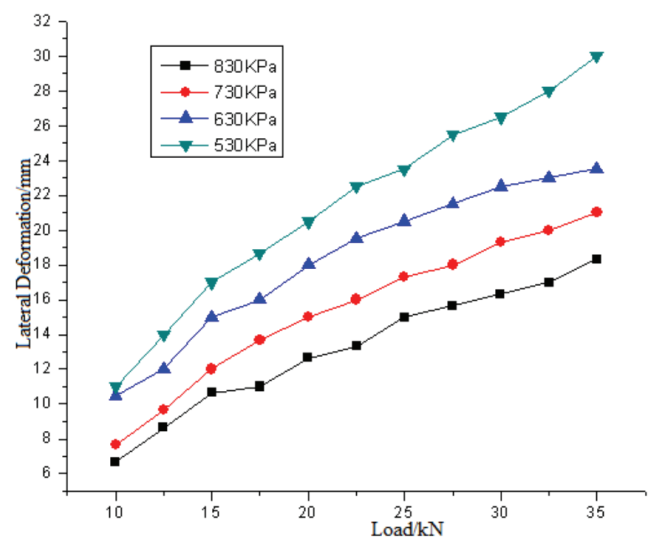

(b) Lateral Deformation

Figure 8 Deformation of New Tire under Different Conditions

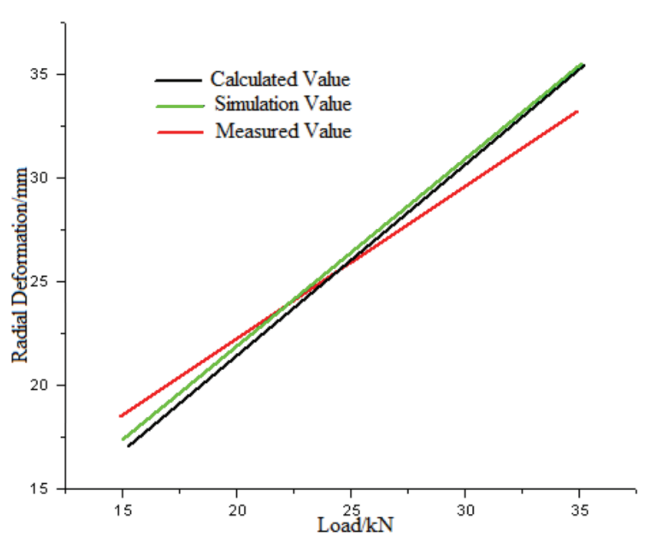

(a) $530 \mathrm{kPa}$

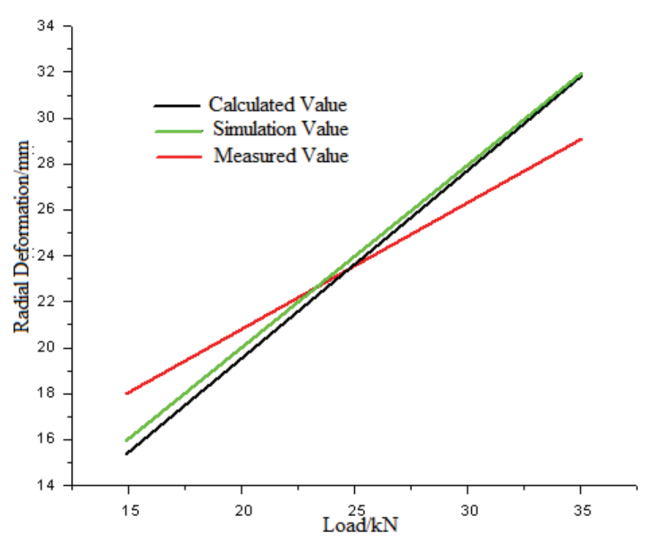

(b) $630 \mathrm{kPa}$ 


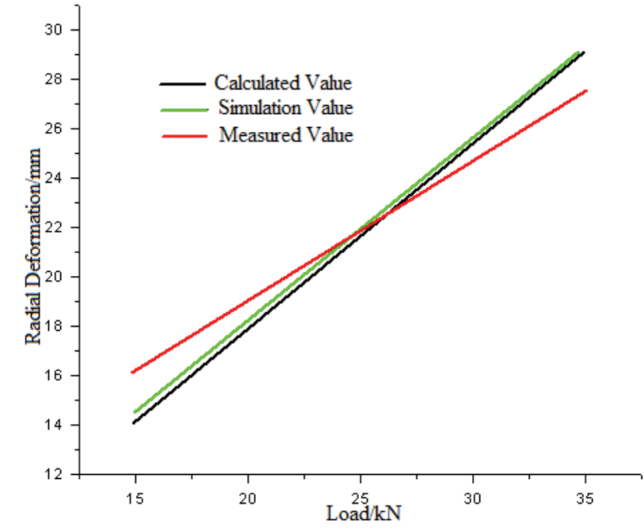

(c) $730 \mathrm{kPa}$

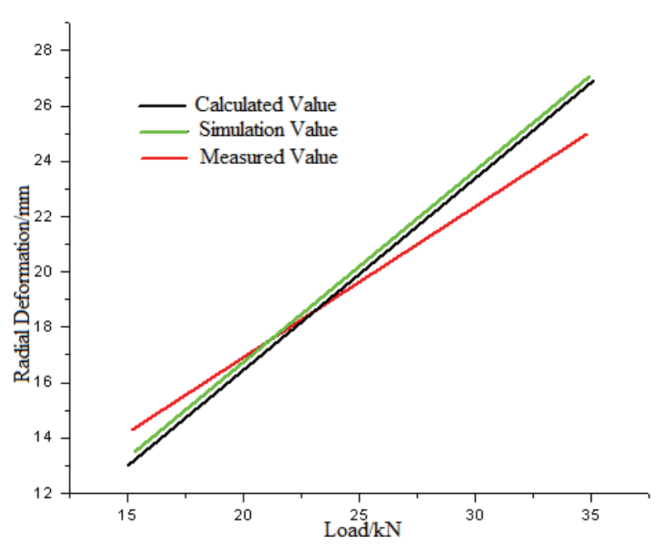

(d) $830 \mathrm{kPa}$

Figure 9 Comparison of Calculated Value, Simulation Value and Measured Value of Retreaded Tire Radial Deformation

Table 1 Regression Equation of Radial Deformation

\begin{tabular}{cl}
\hline Tire Pressure $(\mathrm{kPa})$ & \multicolumn{1}{c}{ Regression Equation } \\
\hline 830 & $\delta_{1}=1.21+8.36 \mathrm{~W}$ \\
730 & $\delta_{2}=0.86+8.46 \mathrm{~W}$ \\
630 & $\delta_{3}=0.59+8.76 \mathrm{~W}$ \\
530 & $\delta_{4}=0.34+8.96 \mathrm{~W}$ \\
\hline
\end{tabular}

The principle of multiple linear regression of the least square method is utilized to propose the formula for calculating the radial deformation theory of 11.00R22.5 retreaded tire as shown in (2).

$\delta=\mu \frac{W^{a_{2}}}{p_{i}^{a_{1}}}$

The logarithm $\ln \delta=\ln \mu+\alpha_{2} \ln W-\alpha_{1} \ln p_{i}$ is obtained at both sides, the theoretical formula for calculating the radial deformation of 11.00R22.5 retreading radial tires is shown in (3).

$\delta=9.46 \times 10^{-3} \frac{W^{0.78}}{p_{i}^{0.467}}$

The formula (3) can be used for calculating the radial deformation value of 11.00R22.5 retreaded radial tires under radial load. It can provide theoretical basis for the research on bearing deformation characteristics of 11.00R22.5 retreaded tires. Figure 10 and figure 11 respectively show the comparison curves of load radial deformation and ground area under different inflation pressures of 11.00R22.5 retreaded tires and new tire of the same brand and model. As can be seen from figure 10 and figure 11, the radial deformation and ground area characteristics of retreaded tire are consistent with that of new tire of the same brand and model, but there are some differences. The radial deformation and ground area change laws of new tires and retreaded tires are linearly regressed when the tire pressure is $830 \mathrm{kPa}$. The radial deformation and ground area regression equations of retreaded tires and new tires are shown in table 2 , and the regression comparison was shown in figure 12 and figure 13. Figure 12 and figure 13 show that the curve slope of retreaded tires is smaller than that of new tires when the tire pressure is constant. The radial deformation of retreaded tires is similar to that of new tires when the load is less than $20 \mathrm{kN}$. Moreover, The difference thereof is larger and larger with the continuous increase of load. It is obvious that the radial stiffness of retreaded tires is larger than that of new tires when it is close to the standard load. The lateral deformation of retreaded tires is smaller than that of new tires when the tire pressure is constant. There is little difference between retreaded tires and new tires when the tire pressure is low. The difference is larger and larger when the tire pressure is gradually increased. The ground area of retreaded tires is smaller than that of new tires when the tire pressure is constant.The difference between retreaded tires and new tires is small when the load is small. The imprinted area of retreaded tires is increased with the increase of vertical load when the tire pressure, lateral force and camber angle used in the test are zero. The imprinted area of retreaded tires is increased with the increase of vertical load. The tire will be approximately elliptic in contact with the ground for the entire width when the vertical load is increased to a certain value. The vertical load is increased continuously, the tire imprint width remains constant, the imprint length is increased, the imprint area is increased, and the imprint is approximately rectangular. The imprint law of new tires is consistent with that of retreaded tires, but the imprinted area of retreaded tires is smaller than that of new tires under the same working condition ${ }^{[6]}$. 


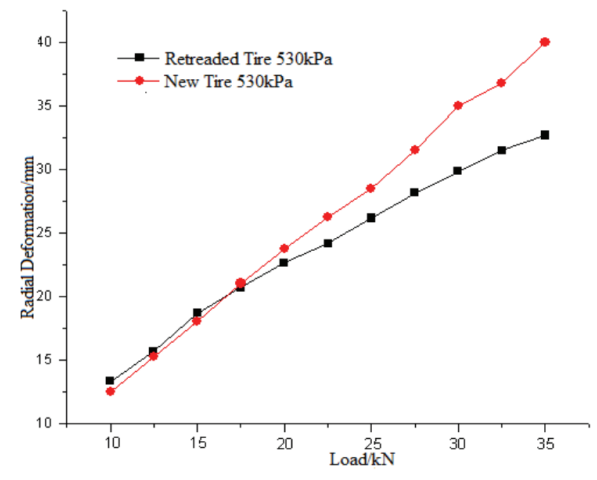

(a) $530 \mathrm{kPa}$

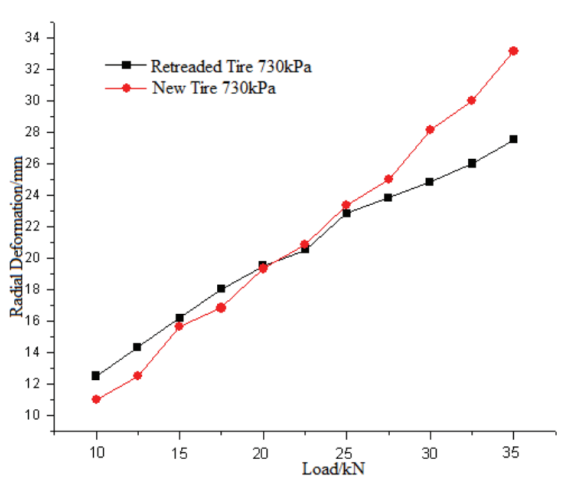

(c) $730 \mathrm{kPa}$

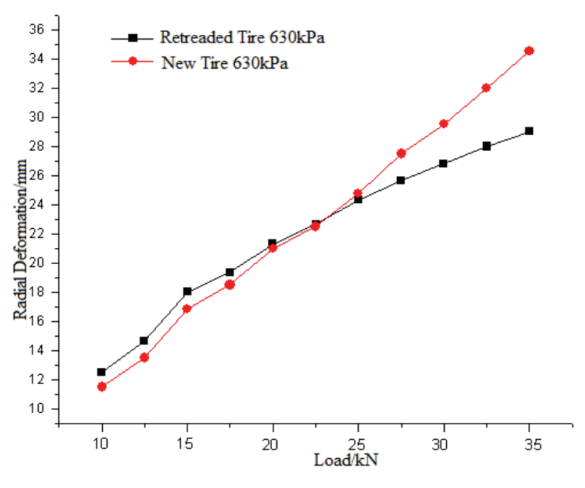

(b) $630 \mathrm{kPa}$

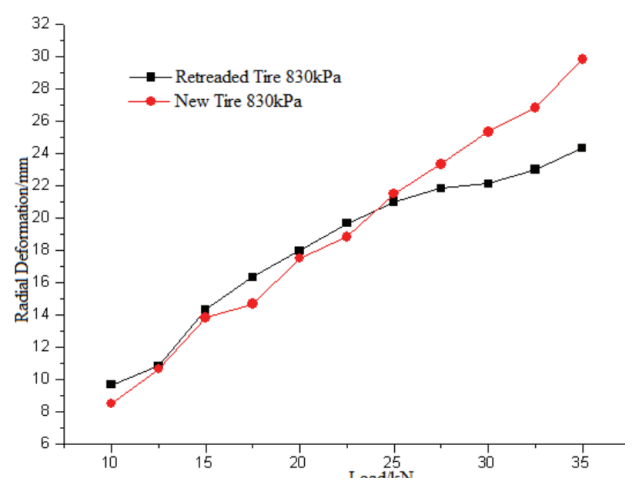

(d) $830 \mathrm{kPa}$

Figure 10 Radial Deformation Contrast of Retreaded Tire with New Tire

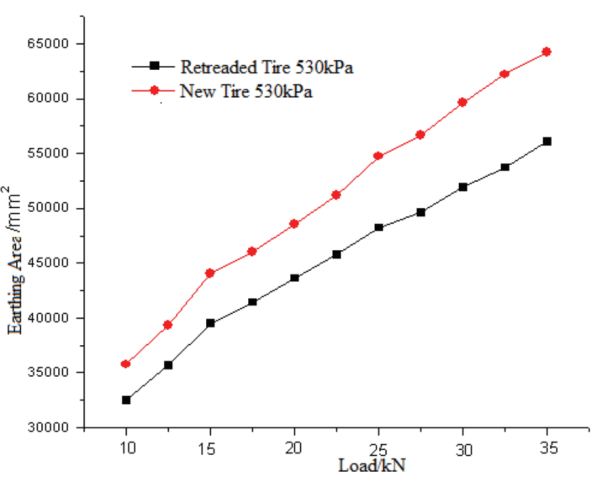

(a) $530 \mathrm{kPa}$

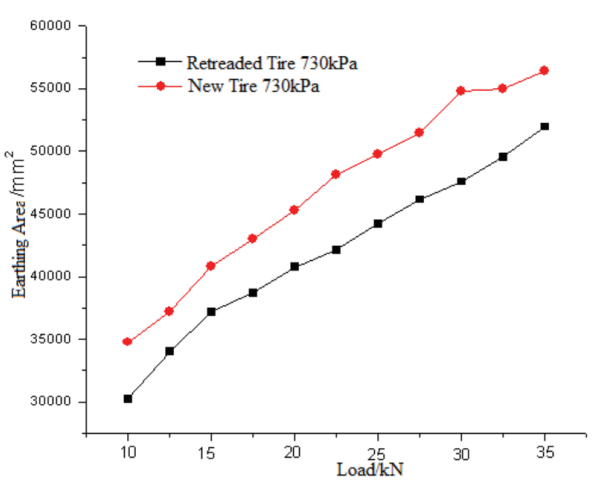

(c) $730 \mathrm{kPa}$

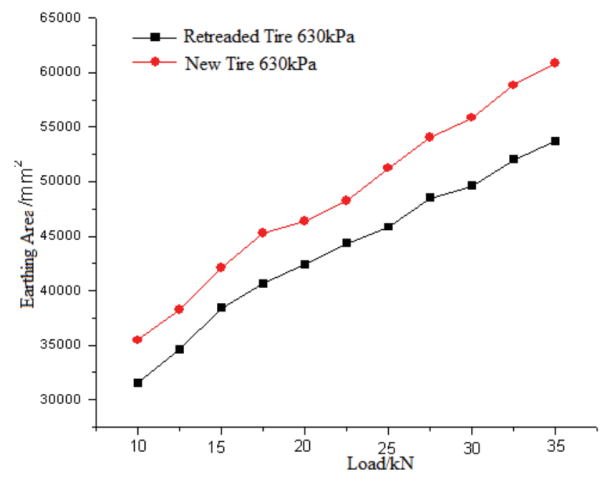

(b) $630 \mathrm{kPa}$

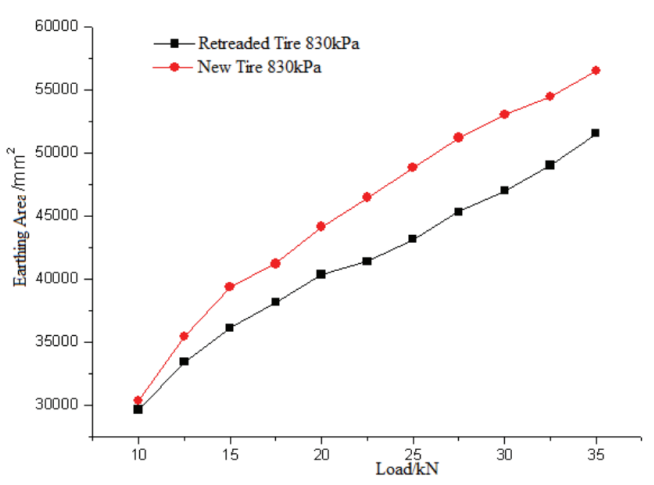

(d) $830 \mathrm{kPa}$

Figure 11 Earthing Area Contrast of Retreaded Tire with New Tire 
Table 2 Radial Deformation and Earthing Area Regression Equations of Retreaded Tire with New Tire

\begin{tabular}{ccc}
\hline & Radial Deformation & Earthing Area \\
\hline Retreaded Tire & $\delta_{1}=4.18+6.38 \mathrm{~W}$ & $S_{1}=-7856+9.67 \mathrm{~W}$ \\
New Tire & $\delta_{2}=1.52+10.24 \mathrm{~W}$ & $S_{2}=2338+12.24 \mathrm{~W}$ \\
\hline
\end{tabular}

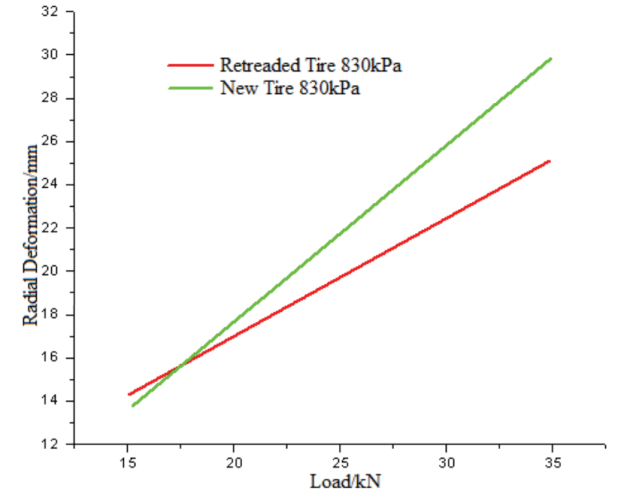

Figure 12 Radial Deformation Regression Contrast of Retreaded Tire with New Tire

\section{Main conclusions}

The radial and lateral deformation of retreaded tires and new tires is increased with the increase of radial load when the tire pressure is constant, and the increase trend is approximately linear. Retreaded tire carcass suffers from certain rubber aging. The overall elastic modulus of the carcass thereof is larger than that of new tires. The waste tire rubber elastic modulus is increased with the increase of aging time according to approximate linear law. The inflation pressure and the steel cord layer in the carcass mainly support the tires during low load, and steel cord is less affected by the aging degree. Therefore, radial stiffness difference is not significant between retreaded tires and new tires. The inflation pressure and carcass steel cord/ rubber composite integrity mainly support the tires when the value is close to the standard load $(30 \mathrm{kN})$. The elastic modulus of retreaded tires is increased due to aging, and its radial stiffness is greater than that of new tires. Moreover, the radial deformation difference between retreaded tires and new tires is increased with the increase of the load. The research method in the paper also can be used for predicting the aging degree of retreaded tire carcass rubber accordingly. Namely the ground area difference is larger if the radial deformation difference between retreaded tires and new tires is larger under near-standard load. The retreaded tire carcass rubber has higher aging degree if the lateral deformation difference between retreaded tires and new tires is lager when the value is close to the standard inflation pressure, and the remaining service life of retreaded tires is shorter.

Author Contributions: Qiang WANG (1981-), Male, Associate professor, Ph.D., Postdoctoral, Master's Supervisor. The main research direction is vehicle tire technology, automobile product optimization design and

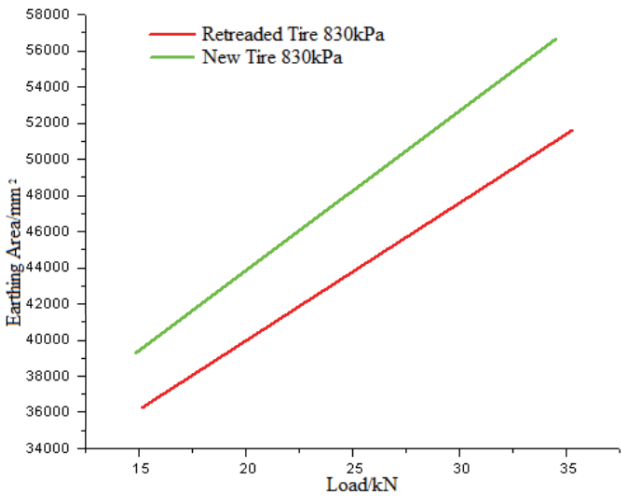

Figure 13 Earthing Area Regression Contrast of Retreaded Tire with New Tire

analysis. E-mail: 630702666@qq.com; Li JIANG, Female, born in 1981, Associate professor, Ph.D., The main research direction is vehicle tire technology.

Acknowledgments: This study was supported by Basic Scientific Research Operating Expense Funding Project of Provincial Univeristies in Heilongjiang Province (2018CX07); Heilongjiang Institute of Engineering Ph. D. Fund. (2016BJ02).

\section{References}

[1] Behnke R, Wollny I, Hartung F, et al(2019). Thermomechanical finite element prediction of the structural long-term response of asphalt pavements subjected to periodic traffic load: Tire-pavement interaction and rutting. Computers and Structures218.

[2] Cui L(2019). Performance Characteristics and Quality Inspection of Retreaded Tire Products. Comprehensive Utilization of Tire Resources in China (12): 41-48.

[3] Liu D(2016). Tire Innovation Leads the Future of Low Carbon and Energy Saving. Modern Rubber Technology42 (02): 9-14.

[4] Mehdi R, Mir Hamid Reza G, Ghasem N(2019). Computer simulation of tire rolling resistance using finite element method: Effect of linear and nonlinear viscoelastic models. Proceedings of the Institution of Mechanical Engineers, Part D: Journal of Automobile Engineering233(11).

[5] Payam F,Abdollah G,Ahmad S Malvajerd,et al(2019). Finite element modeling of the interaction of a treaded tire with clay-loam soil. Computers and Electronics in Agriculture162.

[6] Qi X, Wang Q, Yu J(2010). Load Bearing Deformation Characteristics of Retreaded Tires. Journal of Transportation Engineering10 (05): 47-56. 
[7] Qi X(2010). Load Bearing Simulation and Failure Mechanism Research of Retreaded Heavy Vehicle Tire. Harbin: Northeast Forestry University.

[8] Ravivat R,Chakrit S(2019). Proper Radial Spokes of NonPneumatic Tire for Vertical Load Supporting by Finite Element Analysis. International Journal of Automotive Technology20(4).

[9] Seongrae K, Hanseok P, Byungwoo M, et al(2018). The prediction methodology for tire's high speed durability regulation test using a finite element method. International Journal of Fatigue118.

[10] Wu J, Chen L, Wang Y,et al(2019). Effect of temperature on wear performance of aircraft tire tread rubber. Polymer Testing79.

[11] Wang Q, Jiao S(2018). Load Bearing Deformation Characteristics of Retreaded tires for Engineering Vehicles. Journal of China Highway31 (05): 191-198.

[12] Wang Q, Qi X, Wang Y(2018). Research on Deformation Characteristics of Engineering Retreaded Tire under Free
Inflation and Free Rotation Conditions . Journal of Hubei University of Automotive Industry32 (02): 17-21,26.

[13] Wang Q(2015). Study on Mechanical Properties and Performance Reinforcement Mechanism of Retreaded tires. Harbin: Northeast Forestry University.

[14] Wang Y(2018). Major Problems to Be Solved in The Field of Tire Retreading. Comprehensive Utilization of Tire Resources in China (07): 35.

[15] Xiao J(2017). Tire Retreading and Remanufacturing Technology. Comprehensive Utilization of Tire Resources in China (01): 26-28.

[16] Zhang Y, Gao J, Li Q(2019). Study on tire-ice traction using a combined neural network and secondary development finite element modeling method. Concurrency and Computation: Practice and Experience31(9).

[17] Zhong J(2016). Necessity Analysis of Quality Monitoring of Retreaded Tire Products. China New Technology and New Products(11): 161 